\title{
Low Molecular Weight Antioxidants (LMWA) and their Orchestration
}

\author{
Lamichhane A, ${ }^{1}$ Chaudhary $V^{1}$ Sah $\mathrm{NK}^{1}$, Singh M, ${ }^{2}$ Pandey ${ }^{3},{ }^{3}$ Aggarwal SK ${ }^{1 *}$ \\ ${ }^{1}$ Department of Biochemistry, MM Medical College and Hospital, Solan, India. ${ }^{2}$ Department of Biochemistry, \\ PDM Dental College and Research Institute, Bahadurgarh, India. ${ }^{3}$ Department of Biochemistry, \\ MM Institute of Medical Sciences and Research, Ambala, India
}

\author{
*Corresponding Author: \\ Dr. S.K. Aggarwal \\ Professor and Head, \\ E-mail: drsurinderkaggarwal@gmail.com \\ MM College and Hospital, \\ MM University, Solan (HP), India \\ E-mail: drsurinderkaggarwal@gmail.com
}

\section{Citation}

Lamichhane A, Chaudhary V, Sah NK, Singh M, Pandey $R$, Aggarwal SK. Low Molecular Weight Antioxidants (Imwa) and their Orchestration. Nepal Journal of Medical Sciences 2013;2(2):171-80.

\begin{abstract}
Every day we hear more and more about free radicals and how they are linked with innumerable diseases and health conditions from ageing to muscular degeneration and even some forms of cancer. The problem is not in knowing that these microscopic enemies exist. We know that they do! The problem is how to fight them so that they are rendered harmless. Under normal metabolic conditions each cell of human body is exposed to about $10^{10}$ molecules of superoxide anions (primary free radical) each day. For a person weighing 150 pounds, this amounts to about 4 pounds of superoxide per year, a substantial amount! Once formed, superoxide can react through catalytic pathways in the cell to form many other reactive oxygen/nitrogen species (ROS/RNS). The antioxidant defense system in the human body is extensive and consists of multiple layers, which protect against different types of ROS/RNS. Many of the biological effects of antioxidants appear to be related to their ability not only to scavenge the deleterious free radicals but also to modulate cell signalling pathways.
\end{abstract}

Key words: Diseases; free radicals; low molecular weight antioxidants; ROS/RNS Scavengers

\section{Introduction:}

Continuous exposure to various types of oxidative stress from numerous sources (endogenous and exogenous) has led this cell and the entire organism to develop defense mechanisms for protection against oxygen centered reactive metabolites (ROS/RNS). Indirect approaches may involve control of the endogenous production of ROS/RNS by, for example, altering the activity of enzymes, which indirectly produce oxygen metabolites. An efficient repair system, one of the most important methods for the organism to cope with oxidative damage, consists of enzyme and small mol- ecules that can efficiently repair an oxidative damage site on the macromolecules. Physical defense of biological sites such as membranes is also an important mechanism along the cell to cope with oxidative stress. Among the various defense mechanisms, the one involving antioxidants (enzymic and nonenzymic) are extremely important due to its direct removal of ROS/RNS. The antioxidant defense system apparently developed throughout the evolutionary process, perhaps in response to changing concentration of oxygen in atmosphere. This system contains two major groups-antioxidant enzymes and low molecular weight antioxidants (LMWA). ${ }^{1}$ 
An antioxidant can be defined as: "any substance that when present in low concentrations compared to that of an oxidizable substrate significantly delays or inhibits the oxidation of cellular biomolecules (lipids, proteins, carbohydrates and DNA)". ${ }^{2}$ Antioxidants are a heterogeneous family of molecules, difficult to classify by common shared structural properties. Moreover, other compounds should also be considered that do not act directly as antioxidants, but just indirectly either by modulation of direct agents or by regulation of biosynthesis of antioxidants, promoting their synthesis and/or availability. Several classifications have been attempted in the past taking into account the origin (natural or synthetic), nature (enzymatic or nonenzymatic), chemical-physical properties (hydrophilic or lipophilic), structure (carotenoids, flavonoids, polyphenols, etc.), mechanisms (interceptive, preventive, chain breaking, repairing and adaptive etc; Fig.1). However, antioxidants can be broadly classified as illustrated in Figure 2. In the present review, we aim to highlight the physiological role of low molecular weight antioxidants and their orchestration in the neutralization of ROS/RNS.

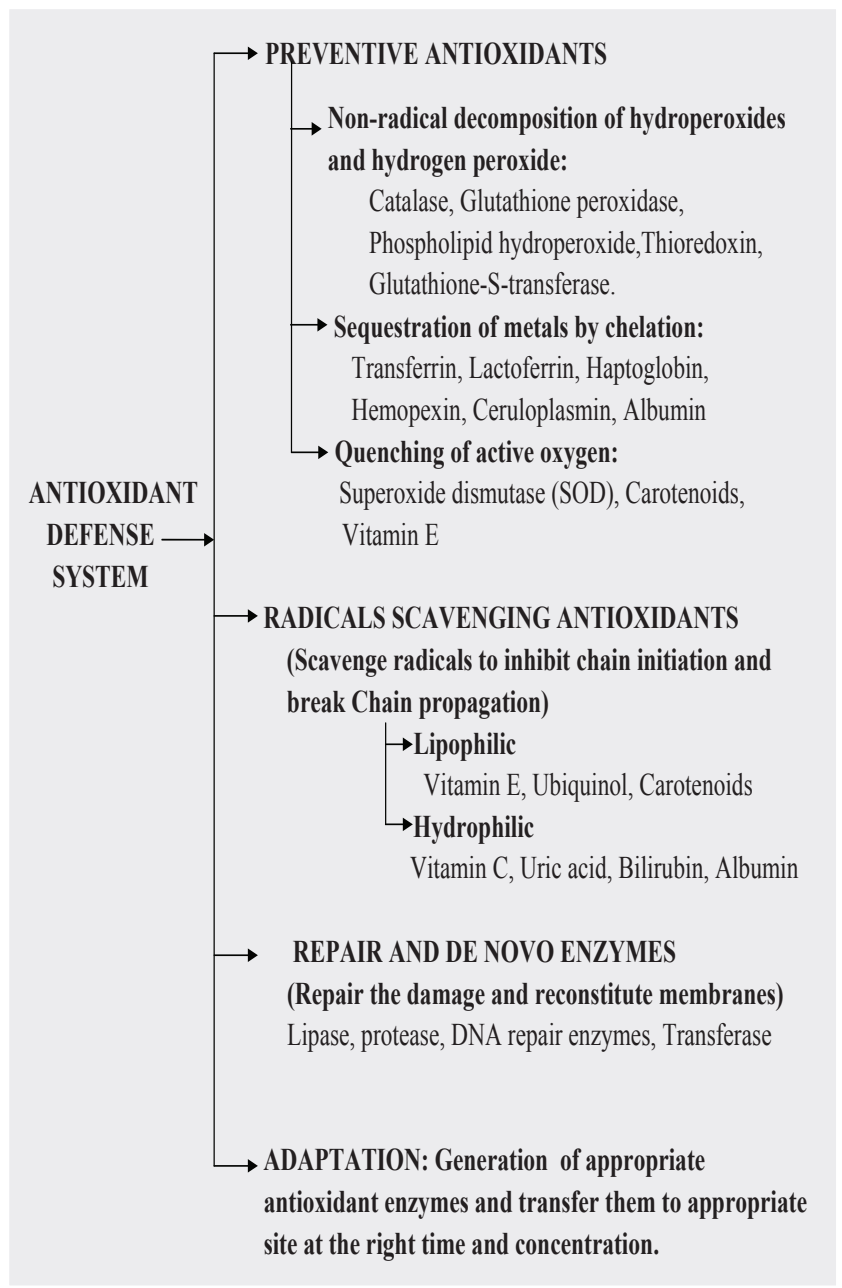

Figure 1: Antioxidant Defense System.

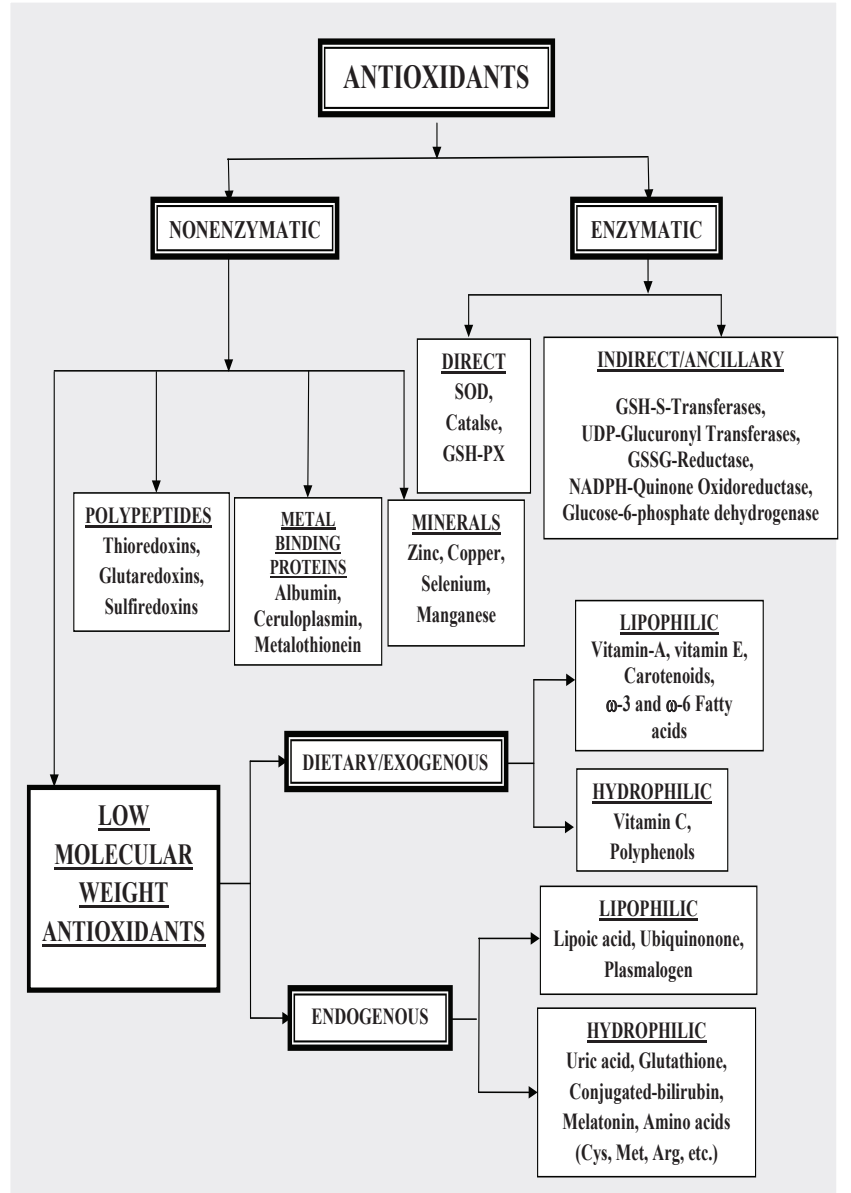

Figure 2. Classification of Antioxidants

\section{LOW-MOLECULAR WEIGHT ANTIOXIDANTS}

There are several low molecular Weight antioxidants (LMWA) that are particularly important in blood and other biological fluids, where antioxidant enzymes are absent or present only in small amounts. The small molecule antioxidants include lipid soluble and water soluble antioxidants. The lipid soluble antioxidants are localized in the cellular membranes and lipoproteins, whereas water-soluble antioxidants are present in aqueous fluids, such as blood and the fluids within the cells and surrounding them. They can scavenge ROS before they cause damage to the various biological molecules, or interrupt the chain reaction of lipid peroxidation. In human tissues, cellular LMWAs are obtained from various sources. ${ }^{4}$

\section{VITAMINS}

\section{Vitamin A}

Vitamin A is a group of nutritionally unsaturated hydrocarbons which include retinol, retinal, retinoic acid and several provitamin A carotenoids among which $\beta$-carotene is the most important. ${ }^{5}$ Plant carotenoids are the primary dietary source of provitamin-A worldwide, with $\beta$-carotene as the 
most well-known provitamin A carotenoid.In vertebrates, $\beta$-carotene is converted into two molecules of retinal, in a reaction catalyzed by $\beta, \beta$-carotene- 15,15 '-monooxygenase (BCMO I). Of the 50 different carotenoids that can be metabolized into vitamin $\mathrm{A}, \beta$-carotene has the highest provitamin A activity. The formed retinal is further metabolized to the vitamin A derivatives retinoic acid (RA) and retinol. ${ }^{6}$

The antioxidant action of carotenoids involves the ability of these pigments to physically quench or inactivate ${ }^{1} \mathrm{O}_{2}$. In doing so, ground state oxygen and triplet state of carotenoid is generated. The triplet state of carotenoid returns to ground state by dissipating its energy through rotational and vibrational interactions with the solvent system. ${ }^{7}$ The efficacy of carotenoids for physical quenching depends upon the number of conjugated bonds that determines their lowest triplet energy state, presence of the functional groups in the molecule and the type of solvent used. ${ }^{8,9}$ Carotenoids are also shown toreact with other free radicals including chain-propagating $\mathrm{RO}_{2}$ 'which are generated in the process of lipid peroxidation. The reaction with free radicals is shown to act by three pathways: radical addition, electron transfer or hydrogen abstraction depending upon the physical property of the carotenoids, type of reactive species and the environment (polar or non-polar) employed to study this reactions. ${ }^{10}$

Vitamin A presents both kinds of antioxidant activities: It is able to scavenge ROS by a direct mechanism and also inhibits, by its oxidized metabolite retinoic acid, NO production through inhibition of iNOS gene transcription in different tissues. $^{3}$ Additionally, the recent report by World Cancer Research Fund (WCRF) ${ }^{11}$ concluded that foods containing carotenoids probably protect against mouth, pharynx, larynx and lung cancer; while foods containing $\beta$-carotene and lycopeneprobably protect against oesophagus and prostate cancer respectively.

\section{Vitamin E/ $\alpha$-Tocopherol}

Vitamin $\mathrm{E}$ is a generic name foreight different isoforms with biological activity, isolated from plantsources: $\alpha-, \beta-, \delta-\gamma$ tocopherol and $\alpha-, \beta-\delta$ - and $\gamma$ - tocotrienol. ${ }^{12}$ Vitamin $\mathrm{E}$ is the primary lipophilic antioxidant,which may have an important role in scavenging of free oxygen radicals and stabilizes the cell membranes. VitaminE is present in all cellular membranes where it may be bound to the protein complexes and may affect oxidative changes which occur in organelles. It acts by at least two different mechanisms: directly scavenges ROS and upregulates antioxidant enzymes such as Glutathione peroxidase, Catalase from liver, Super oxide dismutase, Glutathione-s-transferase, Glutathione reducatse and $\mathrm{NAD}(\mathrm{P}) \mathrm{H}$ : quinine reductase. ${ }^{7}$ Moreover, it is known that antioxidants such as vitamin E, coenzyme Q, ascorbic acid, glutathione and selenium may act synergically, preventing lipid peroxidation and cell destruction. ${ }^{13}$

Vitamin C/Ascorbic acid

Ascorbate is the most important antioxidant present in the hydrophilic compartments. Its activity is expressed through a variety of mechanisms, which still await to be fully clarified. In principle, it acts by scavenging ROS directly, and among these species probably the most important are $\mathrm{O}_{2}$. and ONOO $\cdot{ }^{14}$ Secondly, it has been shown that ascorbate can recycle $\alpha$-tocopherol which in turn helps to prevent lipid peroxidation. ${ }^{15}$ Without ascorbate, the $\alpha$-tocopheroxyl radical can assume a prooxidant role and continue or even enhance the chain reaction of lipid peroxidation. ${ }^{16}$ Further, ascorbate helps in transportation of drugs across the blood brain barrier. ${ }^{17}$ In these regard, it may act as a double targeting molecule, working both as a carrier and as antioxidant in neurodegenerative diseases.

Ascorbate functions as an antioxidant by giving upto two electrons. It can lose one electron to form the semidehydroascorbate, the ascorbyl radical, a relatively stable resonance-stabilized radical of low reactivity. The loss of a second electron results in the formation of dehydroascobate. Ascorbate and ascorbyl radical have low reduction potentials putting these species on the lower end of the "pecking order" and they can therefore react with stronger oxidizing species such as $\mathrm{OH}, \mathrm{O}_{2}^{--}$, etc. Ascorbate plays an important role with the lipophilic antioxidant vitamin $\mathrm{E}$ in protecting the membranes from oxidative stress. Being soluble in the aqueous phase it can regenerate vitamin $\mathrm{E}$ which is present in the membrane. It can reduce the tocopheryl radical, formed when vitamin $\mathrm{E}$ scavenges a lipid radical within a membrane. The hydroxyl group of vitamin $\mathrm{E}$ has been shown to be at the membrane-water interface, in close proximity to the water soluble ascorbate. The tocopherol radical formed from the lipid radical can then be recycled back to tocopherol by ascorbate. ${ }^{18}$

An important aspect of the antioxidant capability of ascorbate is to be recycled back to the reduced ascorbate. Glutathione is important in the recycling of ascorbate by direct chemical reduction and by glutathione dependent enzymes. ${ }^{19}$ Two of the ascorbyl free radicals formed can spontaneously dismutate, generating one molecule of ascorbic acid and one molecule of dehydroascorbic acid. ${ }^{20}$ The latter can be efficiently reduced to ascorbic acid by thioredoxin (Trx) or glutaredoxin (Grx). Glutaredoxins are oxidized by substrates, 
and reduced non-enzymatically by glutathione. In contrast to Trx, which are reduced by thioredoxin reductase, no oxidoreductase exists that specifically reduces Grx. Instead, Grx are reduced by the oxidation of glutathione. Oxidized glutathione is then regenerated by glutathione reductase. ${ }^{21}$ Almost all forms of ROS oxidise methionine residues of proteins to a mixture of the $\mathrm{R}$ - and $\mathrm{S}$-isomers of methionine sulfoxide. Because organisms contain methionine sulfoxide reductases (MSRs), which catalyze the thioredoxin-dependent reduction of sulfoxide back to methionine, the cyclic oxidation/reduction of methionine residues might serve as antioxidant systems to scavange ROS, and also to facilitate the regulation of critical enzyme activities. ${ }^{22}$

\section{ANTIOXIDANTS CONTAINING SULFYDRYL MOI- ETY}

\section{Glutathione (GSH)}

Glutathione, the most abundant tripeptide thiol ( $\gamma$-glutamyl-cysteinyl-glycine, GSH)isa major component of the cellular antioxidant system, with the following characteristics: i) dietary GSH can be partly absorbed from the small intestine and can be synthesized de novo, so that GSH is an exogenous and endogenous antioxidant.ii) although glutathione radical $\left(\mathrm{GS}^{*}\right)$ formed from the oxidation of GSH is a pro-oxidant radical, $\mathrm{GS}^{*}$ can react with another GS* to yield GSSG, which is then reduced to GSH by the NADPH-dependent glutathione reductase; iii) GSH can react with a variety of xenobiotics (electrophilic compounds) in the catalytic reaction of glutathione-S-transferase; iv) GSH effectively scavenges ROS (e.g., lipid peroxyl radical, peroxynitrite and $\mathrm{H}_{2} \mathrm{O}_{2}$ ) and directly and indirectly through enzymatic reactions; v) GSH can conjugate with $\mathrm{NO}$, resulting in the formation of a S-nitroso-glutathione adduct, which is cleaved by the thioredoxin system to release GSH and NO*; and vi) GSH interacts with glutaredoxin and thioredoxin (thiol-proteins), which play important roles in the regulation of cellular redox homeostasis. ${ }^{23}$

Glutathione also participates in redox signalling through the removal of $\mathrm{H}_{2} \mathrm{O}_{2}$, which has the properties of a second messenger, and by reversing the formation of sulfenic acid, a moiety formed by reaction of critical cysteine residues in signalling proteins with $\mathrm{H}_{2} \mathrm{O}_{2}$. Moreover, the concentrations of ascorbate and glutathione are greatly modified in response to a variety of environmental triggers, particularly those that cause increased oxidative stress. GSH, which has one cysteine, and the small protein thioredoxin, which has two cysteines in its active site, often have complementary, if not overlapping roles in cytoprotection. The role of GSH as a substrate for Glutathione peroxidase, to yield GSSG, represents the predominant mechanism for reduction of $\mathrm{H}_{2} \mathrm{O}_{2}$ and lipid hydroperoxides. Therefore, although it does not react directly with hydroperoxides, it works effectively as an antioxidant. Another role for GSH in antioxidant defence depends upon its ability to react with carbon radicals, acting in a concerted manner with SOD to prevent oxidative damage. $^{3}$

\section{Lipoic acid}

$\alpha$-Lipoic acid, which plays an essential role in mitochondrial dehydrogenase reactions, has recently gained considerable attention as an antioxidant. Lipoate, or its reduced form, dihydrolipoate, reacts with $\mathrm{ROS}$ such as $\mathrm{O}_{2}^{--}, \mathrm{OH}, \mathrm{HOCl}, \mathrm{RO}_{2}$. and ${ }^{1} \mathrm{O}_{2}$. It also protects membranes by interacting with vitamin $\mathrm{C}$ and glutathione, which may in turn recycle vitamin $\mathrm{E}$. In addition to its antioxidant activities, dihydrolipoate may exert prooxidant actions through reduction of iron. $\alpha$-Lipoic acid administration has been shown to be beneficial in a number of oxidative stress models such as ischemia-reperfusion injury, diabetes (both $\alpha$-lipoic acid and dihydrolipoic acid exhibit hydrophobic binding to proteins such as albumin, which can prevent glycation reactions), cataract formation, HIV activation, neurodegeneration, and radiation injury. Furthermore, lipoate can function as a redox regulator of proteins such as myoglobin, prolactinand thioredoxin. ${ }^{24}$

\section{MINERALS}

\section{Copper}

Copper attends to important catalytic functions in a number of enzymes such as $\mathrm{Cu}, \mathrm{Zn}-\mathrm{SOD}$, cytochrome oxidase, and ceruloplasmin. In these enzymatic reactions, copper tightly binds to proteins such that the redox activity of the resulting chelate formed is strictly regulated. Copper and zinc, and manganese are indispensable metals for the activities of $\mathrm{Cu}, \mathrm{Zn}-\mathrm{SOD}$ and $\mathrm{Mn}-\mathrm{SOD}$, respectively. Therefore, dietary deficiencies of these minerals markedly decrease tissue $\mathrm{Cu}$, $\mathrm{Zn}-\mathrm{SOD}$ and Mn-SOD activities and result in peroxidative damage and mitochondrial dysfunctions. ${ }^{25}$

\section{Zinc}

Zinc is present in all organs, tissues, and fluids of the body. Out of three types of SOD, Zinc is present in the SOD of cytosol and extracellular space. Although the evidence for the antioxidant properties of zinc is compelling, the mechanisms are still unclear. In general, the mechanism of antioxidation can be divided into acute and chronic effects. Chronic effects involve exposure of an organism to zinc on a long term basis, resulting in the production of other antioxidant 
molecules, such as the metallothioneins. $\mathrm{Zn}^{++}$may induce metallothionein synthesis, forming a zinc-thiolate moiety that functions as a preffered sacrificial site for oxidant attacks. ${ }^{26,27}$ In these terms, zinc acts as a precursor (pro-antioxidant). The acute effects regards either i) protection of protein sulphydryls or ii) reduction of $\mathrm{OH}^{*}$ formation from $\mathrm{H}_{2} \mathrm{O}_{2}$ through the antagonism of redox-active transition metals, such as iron and copper.

\section{Manganese}

The redox-active metal, manganese plays a key role in cellular adaptation to oxidative stress. Without the deleterious side effects of Fenton chemistry, manganese can safely operate as a cofactor for SOD and also provide oxidative stress resistance through formation of nonproteinaceousmanganese-based antioxidant. ${ }^{28}$

\section{Selenium}

The antioxidant effects of selenium appear to be mediated through the glutathione peroxidase that removes potentially damaging lipid hydroperoxides and hydrogen peroxides. Selenium has also been identified as an essential cofactor for selenoprotein $\mathrm{P}$ and other selenoproteins. Thus, selenium can act as an antioxidant in the extracellular space, the cell cytosol, in association with cell membrane preserving structuralintegrity. ${ }^{29}$

\section{AMINO ACIDS AND PEPTIDES:}

\section{Amino acids}

Sulfur containing amino acids like cysteine and methionine have antioxidant effects. Cysteine is the limiting amino acid for GSH synthesis. At low concentrations, the primary mechanism of protection by thiol antioxidants is mediated by their proglutathione property rather than direct scavenging of ROS. Methionine is an efficient scavenger of almost all oxidizing molecules under physiological conditions, such as $\mathrm{H}_{2} \mathrm{O}_{2}, \mathrm{OH}, \mathrm{ONOO}$, and $\mathrm{HOCl}^{30}$

\section{Histidine containing dipeptides}

Carnosine and related dipeptides (anserine, homocarnosine) are naturally occurring histidine containing compounds. They are found in several tissues particularly in muscles. Carnosine is a water soluble natural metabolite of animal tissues. It has antioxidant properties due to its biological functions of scavenging active ROS. It is a scavenger of $\cdot \mathrm{OH}$ and $\mathrm{O}_{2}{ }^{--}$and a strong quencher of ${ }^{1} \mathrm{O}_{2}$. Because of its water solubility, carnosine provides cells with an antioxidant system that functions in the cytosolic environment, where water soluble oxidation mediators (such as transition metals and oxygen radicals) are often present in high concentrations. ${ }^{31}$

\section{Melatonin}

N-Acetyl-5-methoxytryptamine, commonly known as melatonin, is a product of the vertebrate pineal gland as well as of other select organs. It was initially found to function as a mediator of circannual reproductive rhythms as well as of circadian cycles. Subsequently, however, melatonin was shown to have significantly broader actions including oncostatic effects, immune system stimulation and anti-inflammatory functions. Even more recently, and somewhat unexpectedly, melatonin was identified as a powerful direct free radical scavenger and indirect antioxidant. ${ }^{32}$

Melatonin is a powerful free radical scavenger and a broad spectrum antioxidant. Because of its small size and highly lipophilic properties, melatonin crosses all bio membranes and easily reaches subcellular compartments, including mitochondria and nuclei. Melatonin prevents lipid peroxidation, protein, and DNA damage. In particular, melatonin has been found to preserve optimal mitochondrial function and homeostasis by reducing and preventing mitochondrial oxidative stress, thereby curtailing subsequent apoptotic events and cell death. The ability of melatonin to scavenge the ${ }^{\circ} \mathrm{OH}$ is much higher than other antioxidants including mannitol, glutathione and vitamin E. Melatonin can also scavenge $\mathrm{O}_{2}{ }^{--}, \mathrm{OH},{ }^{1} \mathrm{O}_{2}, \mathrm{H}_{2} \mathrm{O}_{2}, \mathrm{HOCl}, \mathrm{NO}+$, and $\mathrm{ONOO}^{-}$. Not only is melatonin itself a direct free radical scavenger, but metabolites that are formed during these interactions (i.e., cyclic 3-hydroxymelatonin, N1-acetyl-N2-formyl-5-methoxykynuramine, and N1-acetyl-5 methoxykynuramine), are likewise excellent scavengers of toxic reactants. Furthermore, melatonin plays an important role in activating antioxidant defenses such as SOD, catalase, glutathione reductase and glucose-6-phosphate dehydrogenase. ${ }^{33}$

\section{Polyamines}

The naturally occurring polyamines, spermine, spermidine and the diamine putrescine are widespread in nature. They have been implicated in growth and differentiation processes. Polyamines also have been reported as antioxidant (free radical scavengers). ${ }^{34,35}$

\section{METABOLIC WASTE PRODUCTS}

\section{Uric acid}

Uric acid is produced from hypoxanthine and xanthine by xanthine oxidase $(\mathrm{XO})$ and xanthine dehydrogenase $(\mathrm{XDH})$ enzymes. ${ }^{36}$ Xanthine dehydrogenase belongs to the group of molybdenum containing hydroxylases involved in the 
oxidative metabolism of purines. The enzyme is a homodimer. Xanthine dehydrogenase can be converted to xanthine oxidase by reversible sulfhydryl oxidation or by irreversible proteolytic modification. At physiological $\mathrm{pH}$, it is present as urate ion since $\mathrm{pKa}$ of uric acid is around 5.4. Ames et al. ${ }^{37}$ suggested that urate is a powerful scavenger of ROS in vitro, proposing that it functions as a biological antioxidant. Uric acid is one of the most important antioxidants in plasma. Urate reacts with ${ }^{\circ} \mathrm{OH}, \mathrm{RO}_{2} \cdot{ }^{1} \mathrm{O}_{2}, \mathrm{H}_{2} \mathrm{O}_{2}, \mathrm{O}_{3}, \mathrm{NO}_{2}{ }^{*}$, $\mathrm{ONOO}^{-}$and oxo-heme oxidants produced by peroxide reaction with haemoglobin. ${ }^{37,38}$ It also binds metal ions like $\mathrm{Cu}$ and Fe making them poorly reactive in catalysing free-radical reactions. ${ }^{30,40}$ These reactions generate urate radical and the unpaired electron is localised over the purine ring, giving a resonance stabilized radical that does not react with oxygen to form $\mathrm{RO}_{2} \cdot 38$

It has been reported that uric acid may also prevent degradation of type $3 \mathrm{SOD}\left(\mathrm{SOD}_{3}\right)$, an enzyme critical in maintaining endothelial and vascular function. The removal of $\mathrm{O}_{2}{ }^{--}$by $\mathrm{SOD}_{3}$ prevents the reaction and inactivation by $\mathrm{O}_{2}^{--}$of the important endothelial vasodilator, $\mathrm{NO} . \mathrm{SOD}_{3}$, by removing superoxide, therefore helps to maintain NO level and hence endothelial function. Normally, $\mathrm{SOD}_{3}$ is inactivated in the presence of $\mathrm{H}_{2} \mathrm{O}_{2}$, suggesting a feedback inactivation of the enzyme. However, uric acid blocks SOD inactivation by $\mathrm{H}_{2} \mathrm{O}_{2}$, by regenerating $\mathrm{SOD}_{3}$ with the production of urate radical. This latter radical, although potentially pro-oxidant, has been found to be markedly less reactive than classic oxidants and can be rapidly regenerated back to urate in the presence of ascorbate. ${ }^{41}$

\section{Bilirubin}

In 2002, an attractive concept was put forward by Snyder and colleagues consisting of the biliverdin reductase (BVR) mediated reduction of biliverdin (BV) to bilirubin (BR) and subsequent oxidation of BR by hydrogen peroxide back to BV forming a catalytic antioxidant cycle that is driven by NADPH, the reducing cofactor of BVR (Fig 3) Baranano et al., 2002 ${ }^{42}$; Greenberg, $2002^{43}$; Sedlak and Snyder, 2004. ${ }^{44}$ Especially lipophilic ROS such as lipid hydroperoxides and peroxyl radicals were described to feed this antioxidant cycle and later, synergistic effects of glutathione have been described (Sedlak et al., 2009) ${ }^{45}$ Many researchers favour an important role of biliverdin reductase for antioxidant and cytoprotective effects of the heme oxygenase system. ${ }^{46-51}$

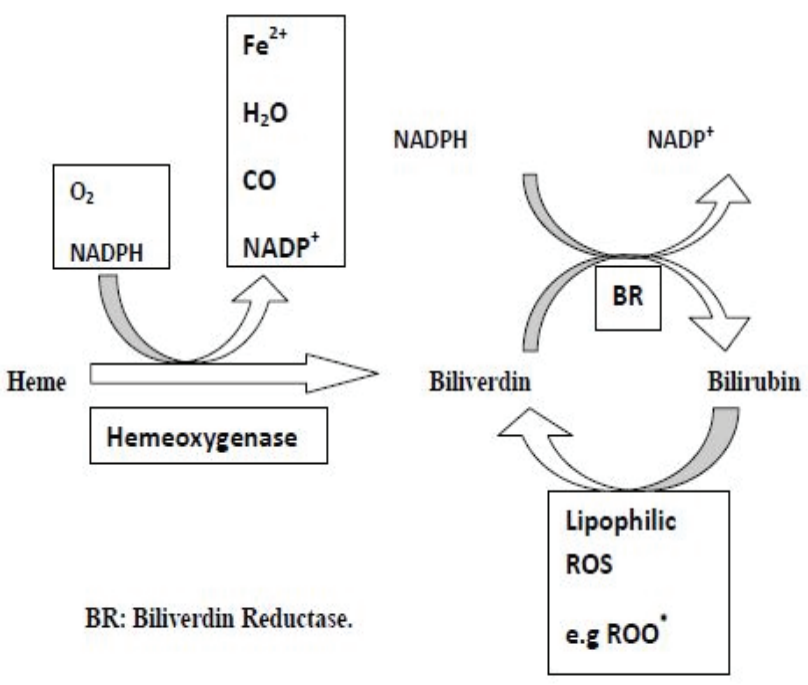

Figure 3: Role of Biliverdin Reductase and Bilirubin as Free Radical Scavengers.

\section{OTHERS}

\section{Coenzyme Q}

Coenzyme Q or ubiquinone is a biological compound that is widely distributed in plants, animals and in most microorganisms. It is a lipophilic molecule present in all tissues and cells and associated with biomembranes. It is composed of a redox active benzoquinone ring conjugated to an isoprenoid chain. The length of the chain differs amongspecies; in humans, ubiquinone contains predominantly 10 isoprenyl units and is designated $\mathrm{CoQ}_{10}{ }^{52}$ This compound acts both as an electron carrier and proton translocator. Due to its lipophilicity, ubiquinone interacts with dehydrogenases and shuttles a pair of two single electrons to cytochromes by diffusion. ${ }^{53}$ Coenzyme $\mathrm{Q}$ is known to play an important role as a mobile redox proton carrier in the energy transducing membranes.Besides, coenzyme Qacts as a carrier of electrons from respiratory complexes I and II to complex III, and generation of $\mathrm{O}_{2}^{--}$by autoxidation of ubisemiquinone. The reduced form of coenzyme $\mathrm{Q}$, ubiquinol, has been shown to act as an antioxidant against free radical mediated oxidations in membranes and lipoproteins. ${ }^{54,55}$

Current evidence suggests that ubiquinol and $\alpha$-tocopherol act in concert to scavenge radicals during autoxidation of mitochondrial membranes. $\alpha$-Tocopherol seems to act as a direct scavenger forming tocopheroxyl radical, whereas ubiquinol reacts with tocopheroxyl radical to regenerate $\alpha$-tocopherol. ${ }^{54}$

\section{Plasmalogens}

Plasmalogens are phospholipids containing vinyl ether linkage at the $s n-1$ position of the glycerophospholipid back- 
bone. They are physiological antioxidants with the vinyl ether functionality serving as sacrificial trap for free radicals and singlet oxygen. The quenching of ${ }^{1} \mathrm{O}_{2}$ by plasmalogens is mostly reactive in nature and results from its preferential interaction with the vinyl ether bond. Broniec et al. suggested that plasmalogens could protect unsaturated membrane lipids against oxidation induced by singlet oxygen, and that the oxidation products are not excessively cytotoxic. ${ }^{56}$

\section{ORCHESTRATION OF LMWA}

The interaction between different LMWA is a complex processes, with various metabolites and enzymes system having synergistic and interdependent effects on one another. The amount of protection provided by any antioxidant therefore depends on its standard redox potentials $\left(\mathrm{E}_{0} ; \mathrm{v}\right)$, concentration, the reactivity towards particular ROS/ RNS, and the status of antioxidants with which it may interact (Table 1). Higher the negative standard redox potential of a LMWA, more is the reducing capacity. Glutathione with its highest negative redox potential renders it an important antioxidant and a convenient cofactor for enzymatic reactions that require readily available electron pairs, the so-called "reducing equivalents".

Table 1: Comparative standard redox potential of some LMWA which participate in the formation of antioxidant network.

\begin{tabular}{ccc}
\hline $\mathbf{E}_{\mathbf{0}}(\mathbf{v})$ & $\begin{array}{c}\text { Low Molecular Weight } \\
\text { Reduced }\end{array}$ & $\begin{array}{c}\text { Antioxidants (LMWA) } \\
\text { Oxidized }\end{array}$ \\
\hline-0.330 & GSH (Glutathione) & GSSG \\
-0.320 & Dihydrolipoic acid & Lipoic acid \\
-0.220 & Ubiquinone & Ubisemiquinone \\
-0.038 & Ubiquinone & Ubiquinol \\
0.190 & Ubisemiquinone & Ubiquinol \\
0.282 & Ascorbate & Ascorbyl \\
0.480 & Tocopherol & Tocopheroxyl \\
\hline
\end{tabular}

Previously, scientists believed that each antioxidant works separately, independently of others. However, Packer and Colman ${ }^{57}$ showed that there is a dynamic connection among certain key antioxidants. The expression "antioxidant orchestration"clearly demonstrates that antioxidants do not act alone but are linked together into a net. The interaction of various LMWA, e.g. DHLA, GSH, ubiquinone, ascorbate and $\alpha$-tocopherol has already been established based on the concept of mode of action of the antioxidant(Fig 4).

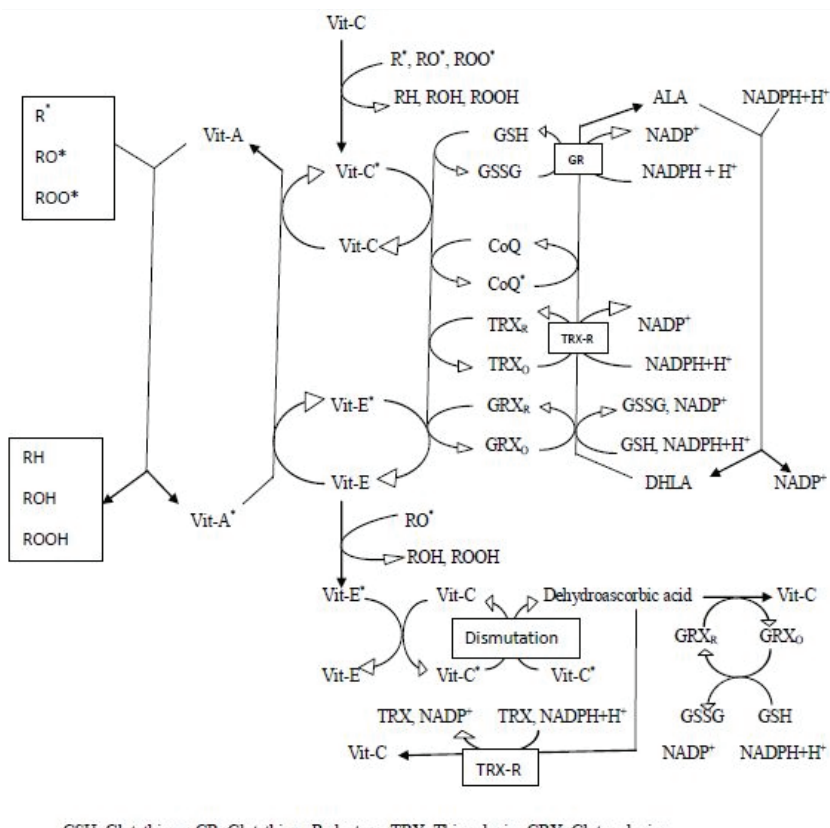

GSH: Glutathione; GR: Glutathione Reductase; TRX: Thioredoxin; GRX: Glutaredoxin

TRX-R. Thioredoxin Reductase; ALA: $\alpha$-Lipoic acid, DHLA: Dihydrolipoic acid $\mathrm{R}^{*}$ : Alkyl Radical; RO': Alkoxyl radical; ROO': Peroxyl radical

Figure 4: Orchestration of Low Molecular Weight Antioxidants

\section{CONCLUSIONS AND FUTURE PERSPECTIVES}

The ROS/ RNS are involved in a variety of biologic phenomena. In lowamounts, they modify and fine-tune intracellular signalling, and their potentially adverse effects are prevented by the various cellular antioxidant systems. Oxidative stress is the result when the ROS/RNS are present in higheramounts due to their excessive production and/or the inadequate antioxidant systems and failure to repair oxidative damage. Oxidative stress is involved in damage of macromolecules such as polyunsaturated fatty acids (PUFA) in membrane phospholipids and plasma lipoproteins, essential proteins and nucleic acids. Additionally, ROS/RNS induced oxidative stress has been implicatedwith age related diseases such as atherosclerosis and cardiovascular diseases, neurodegenerative diseases, cancers, skin diseases, complications in diabetes mellitus etc.

Most of the LMWA act as "double edged swords" as they are beneficial within their limited range and act as pro-oxidants that boost up further oxidant production following excess intake.Antioxidants have become very well recognized nutraceutical ingredients of the diet. Intake of LMWA rich diet or supplements may help in the prevention of various diseases and complications as discussed. The regular screening of antioxidant levels should be done to know the deficient or the excess status. The therapeutic use of anti- 
oxidants might involve naturally occurring molecules (with or without structural adaptation) or completely synthetic products. One possible limitation of antioxidant therapy is that cell death will already have occurred by the time overt symptomatology of disease becomes evident. Therefore, it is important to start the therapies directed against oxidative stress at an early stage in the disease process.

\section{Conflicts of interests: none}

\section{References:}

1. Kohen R, Nyska A. Oxidation of Biological Systems: Oxidative stress phenomena, antioxidants, redox reactions and methods for their quantification. Toxiclogic pathology 2002;30:620-50.

2. Halliwell B, Gutteridge JC. Definition and measurement of antioxidants in biological systems. Free Radic Biol Med 1995;18:125-6.

3. Vertuani S, Angusti A, Manfredini S. The Antioxidants and Pro-oxidants Network: An Overview. Curr Pharm Des 2004;10:1677-94.

4. Kumar S. Free Radicals and Antioxidants: Human and Food System. Adv Appl Sci Res 2011; 2:129-35.

5. Van Bennekum A; Werder M, Thuahnai, ST, et al. Class B scavenger receptor-mediated intestinal absorption of dietary $\beta$-carotene and cholesterol. Biochemistry $2005 ; 44: 4517-25$.

6. Mueller L, Boehm V. Antioxidant activity of $\beta$-carotene compounds in different in vitroassays. Molecules 2011;16:1055-69.

7. Krinsky NI, Johnson EJ. Carotenoid actions and their relation to health and disease. Mol Aspects Med 2005;26:459-516.

8. Di MP, Murphy ME, Sies H. Antioxidant defense systems: the role of carotenoids, tocopherols, and thiols. Am J Clin Nutr 1991 53:194S-200S

9. El-Agamey A, Cantrell A, Land EJ, et al. Are dietary carotenoids beneficial? Reactions of carotenoids with oxy-radicals and singlet oxygen. Photochem Photobiol Sci 2004;3:802-11.

10. Krinsky NI, Yeum KJ. Carotenoid-radical interactions. Biochem Biophys Res Commun 2003; 6:754-60.

11. World Cancer Research Fund/American Institute for Cancer Research. Food, Nutrition, Physical Activity, and the Prevention of Cancer: a Global Perspective.
Washington DC: AICR, 2007.

12. Packer L, Weber SU, Rimbach G. Molecular aspects of alpha-tocotrienol antioxidant action and cell signalling. J Nutr 2001;131:369S-73S.

13. Branka IO, Sladan Z, Pavlovic SD, et al. Protective role of vitamin $\mathrm{E}$ on antioxidant defense system and lipid peroxide concentration in the blood of rats acutely exposed to cadmium. Kragujevac J Sci 2001;23:115-26.

14. May JM. How does ascorbic acid prevent endothelial dysfunction? Free Radic Biol Med 2000;28:1421-9.

15. Diaz MN, Frei B, Vita JA, et al. Antioxidants and atherosclerotic heart disease. N Engl J Med 1997;337: 408-16.

16. Doba T, Burton GW, Ingold KU. Antioxidant and co-antioxidant activity of vitamin $\mathrm{C}$. The effect of vitamin $\mathrm{C}$, either alone or in the presence of vitamin $\mathrm{E}$ or a water soluble vitamin $\mathrm{E}$ analogue, upon peroxidation of aqueous multilamellar phospholipid liposomes. Biochim Biophys Acta 1985;835:298-303.

17. Manfredini S, Pavan B, Vertuani S, et al. Design synthesis and activity of ascorbic acid prodrugs of nipecotic, kyneurenic and diclophenamic acids, liable to increase neurotropic activity. J Med Chem 2002;45:559-62.

18. Butterfield DA, Castegna A, Pocernich CB, et al. Nutritional approaches to combat oxidative stress in Alzheimer's disease. J Nutr Biochem 2002;13:444-61.

19. Sorg O. Oxidative stress: a theoretical model or a biological reality? C R Biol 2004;327:649-62.

20. Jonas N, Elias SJ. Reactive oxygen species, antioxidants, and the mammalian thioredoxin system. Free Radic Biol Med 2001;31:1287-312.

21. Holmgren A, Fernandes AP. Glutaredoxins: glutathione-dependent redox enzymes with functions far beyond a simple thioredoxin backup system. Antioxid Redox Signal 2004;6:63-74.

22. Stadtman ER, Moskovitz J, Berlett BS, et al. Cyclic oxidation and reduction of protein methionine residues is an important antioxidant mechanism. Mol cell Biochem 2002;3:234-5.

23. Palozza P, Serini S, DiNicuolo F, et al. Prooxidant effects of $\beta$-carotene in cultured cells. Mol Aspects Med 2003;24:353-62.

24. Packer L, Witt EH, Tritschler HJ. Alpha-lipoic acid 
as a biological antioxidant. Free_Radic Biol Med 1995; 19:227-50.

25. Videla LA, Fernandez V, Tapia G, et al. Oxidative stress mediated hepatotoxicity of iron and copper: role of Kupffer cells. Biometals 2003;16:103-11.

26. Rostan EF, DeBuys HV, Made DL, et al. Evidence supporting zinc as an important antioxidant for skin. Int $\mathrm{J}$ Dermatol 2002;41:606-11.

27. Onderci M, Sahin N, Sahin K, et al. Antioxidant properties of chromium and zinc: in vivo effects on digestibility, lipid peroxidation, antioxidant vitamins and some minerals under a low ambient temperature. Biol Trace Elem Res 2003;92:139-50.

28. Aguirre JD, Culotta VC. Battles with Iron: Manganese in Oxidative Stress Protection. J Biol Chem 2013;287:13541-8.

29. May SW. Selenium-based pharmacological agents: an update. Expert Opin Investig Drugs 2002;11:1261-9.

30. Atmaca G. Antioxidant effects of sulfur-containing amino acids. Yonsei Med J 2004;45:776-88.

31. Gariballa SE, Sinclair AJ. Carnosine: Physiological properties and therapeutic potential. Age Ageing 2000;29:207-10.

32. Russel JR, Tan DX, Juan CM, et al. Melatonin as an antioxidant: biochemical mechanisms and pathophysiological implications in humans. Acta Biochimica Polonica 2003;50:1129-46.

33. Tamura H, Takasaki A, Taketani T, et al. Melatonin as a free radical scavenger in the ovarian follicle. Endocr $\mathrm{J}$ 2013;60:1-13.

34. Ha HC, Nilantha SS, Kuppusamy P, et al. The natural polyamine spermine functions directly as a free radical scavenger. Proc Natl Acad Sci USA 1998;95:11140-5.

35. Fujisawa S, Kadoma Y. Kinetic Evaluation of Polyamines as Radical Scavengers. Anticancer Res 2005;25:965-70.

36. Becker BF. Towards the physiological function of uric acid. Free Radic Biol Med 1993;14:615-31.

37. Ames BN, Cathcart R, Schwiers E, et al. Uric acid provides an antioxidant defense in humans against oxidantand radical-caused aging and cancer: a hypothesis. Proc Natl Acad Sci USA 1981;78:6858-62.
38. Halliwell B, Gutteridge JM. Free Radicals in Biology and Medicine. Fourth ed. Oxford University Press Inc., New York; 2007.

39. Davies KJ, Sevanian A, Muakkassah-Kelly SF, et al. Uric acid-iron ion complexes. A new aspect of the antioxidant functions of uric acid. Biochem J 1986;235:74754.

40. Ko KM, Godin DV. Inhibition of transition metal ion-catalysed ascorbate oxidation and lipid peroxidation by allopurinol and oxypurinol. Biochem Pharmacol 1990;40:803-9.

41. Ostdal H, Davies MJ, Anderson HJ. Reaction between protein radicals and other biomolecules. Free Radic Biol Med 2002;33:201-9.

42. Baranano DE, Rao M, Ferris CD, et al. Biliverdin reductase: amajorphysiologic cytoprotectant. Proc Natl Acad Sci USA 2002;99:16093-8.

43. Greenberg DA. The jaundice of the cell. Proc Natl Acad Sci U.S.A 2002;99:15837-9.

44. Sedlak TW, Snyder SH. Bilirubin benefits: cellular protection by a biliverdin reductase antioxidant cycle. Pediatrics 2004;113:1776-82.

45. Sedlak TW, Saleh M, Higginson DS, et al. Bilirubin and glutathione have complementary antioxidant and cytoprotective roles. Proc Natl Acad Sci USA 2009;106:5171-6.

46. Young SC, Storm MV, Speed JS, et al. Inhibition of biliverdin reductase increases ANG II-dependent superoxide levels in cultured renal tubular epithelial cells. Am J Physiol Regul Integr Comp Physiol 2009;297:R1546R1553

47. Liu Y, Liu J, Tetzlaff W, et al. Biliverdin reductase, a major physiologic cytoprotectant, suppresses experimental autoimmune encephalomyelitis. Free Radic Biol Med 2006;40:960-7.

48. Panahian N, Huang T, Maines MD. Enhanced neuronal expression of the oxidoreductase biliverdin reductase after permanent focal cerebral ischemia. Brain Res 1999;850:1-13.

49. Ahmad Z, Salim M, Maines MD. Human biliverdin reductase is a leucine zipper like DNA binding protein and functions in transcriptional activation of hemeoxygenase- 1 by oxidative stress. J Biol Chem 2002;277:922632. 
50. Maines MD. Biliverdin reductase: PKC interaction at the cross talk of MAPK and PI3K signaling pathways. Antioxid Redox Signal 2007;9:2187-95.

51. Kim SY, Kang HT, Choi HR, et al. Biliverdin reductase $A$ in the prevention of cellular senescence against oxidative stress. Exp Mol Med 2011;43:15-23.

52. Catarina MQ, Michio H. Coenzyme Q and Mitochondrial Disease. Dev Disabil Res Rev 2010;16:183-8.

53. Nohl H, Gille L, Staniek K. The biochemical, pathiophysiological, and medical aspects of ubiquinone function. Ann NY Acad Sci 1998;854:394-409.

54. Rajindar SS, Michael JF. Coenzyme Q, oxidative stress and ageing. Mitochondrion 2007;7:S103-S111.
55. Niki E. Mechanisms and dynamics of antioxidant dynamics of antioxidant action of ubiquinol. Mol aspects Med 1997;18:63-70.

56. Broniec A, Klosinski R, Pawlak A, et al. Interaction of plasmalogens and their diacyl analogs with singlet oxygen in selected model systems. Free Radic Biol Med 2011;50:892-8.

57. Packer L, Colman C. The antioxidant miracle. John Wiley and Sons, New York, 1999; 1-30. 